\title{
Correspondence
}

Epidemiol. Infect. (2012).

doi:10.1017/S0950268812000441

First published online 26 March 2012

\section{Determinants of campylobacteriosis notifications in New Zealand}

To the Editor:

In the November 2011 issue of Epidemiology and Infection, Spencer et al. reported on factors associated with campylobacteriosis in New Zealand [1]. We conducted a similar analysis in the state of Illinois from 2004 to 2007. During this period, the average Campylobacter rates for Illinois and the USA were 10.7 and 12.7 cases/ 100000 , respectively [2]. Several studies have reported an increase in cases in young adults [2-6], which we also observed in Illinois $(9 \cdot 6$ cases $/ 100000$ in the 20-29 years age group; Fig. 1). We also observed a higher rate of cases in the 50-59 years age group (14.9 cases/ $100000)$.

To further evaluate age groups with increased rates of infection, all cases reported in the 20-29 years $(n=596)$ and $50-59$ years $(n=712)$ age groups were geocoded to the census tract level. Demographic data for each census tract were obtained from the 2000 United States Census. In the adjusted Poisson regression models, census tracts with factors related to higher socioeconomic status (smaller family size, higher median income, higher rates of college degrees, higher percent of white population) and higher percent of population living with relatives were significantly more likely to report cases of Campylobacter in both age groups (Table 1); increased rate of home ownership, another indicator of higher socioeconomic status, was also associated with greater risk in the 20-29 years age group (Table 2).

We did not find a difference between urban and rural populations, as Spencer did; however, they found a rural/urban difference in teenagers, who were not included in this analysis. Cattle farming is uncommon in Illinois and therefore was not examined. Seasonal and sex patterns were similar to those reported by Spencer. As Spencer suggested, this association between Campylobacter and higher socioeconomic status may reflect better access to healthcare, since many cases go undiagnosed, or different eating patterns. We looked briefly at food deserts (areas where access to fresh foods are very

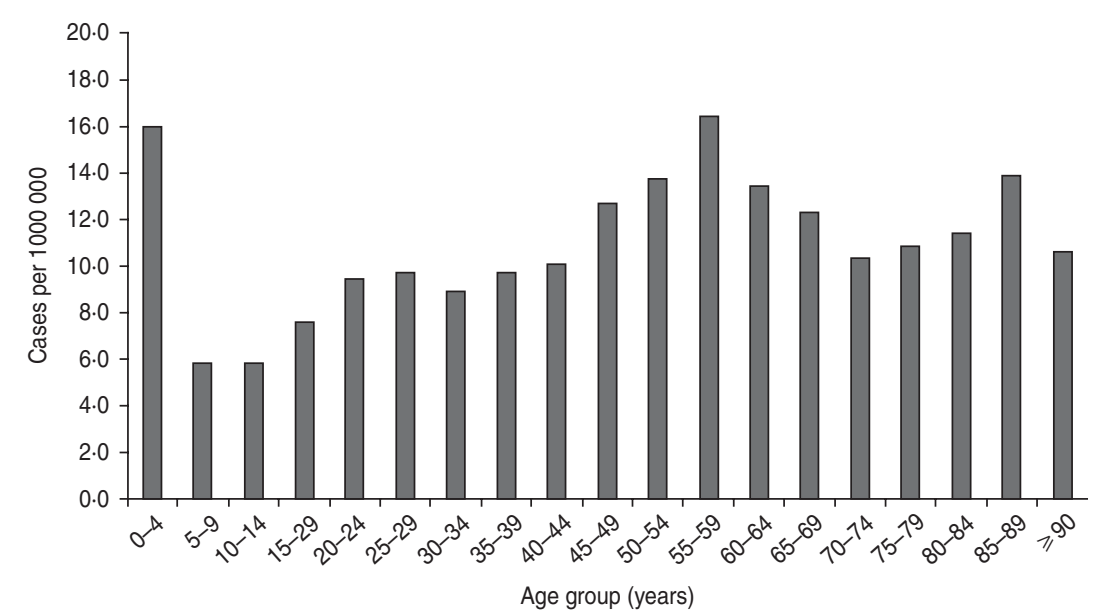

Fig. 1. Average reported Campylobacter case rate in Illinois by age, 2004-2007. 
Table 1. Census tract factors associated with reported cases of Campylobacter in the 20-29 years age group

\begin{tabular}{|c|c|c|}
\hline Variable & $\begin{array}{l}\text { IRR }(95 \% \text { CI }) \text {, } \\
\text { adjusted for all } \\
\text { other variables }\end{array}$ & $P$ value \\
\hline \multicolumn{3}{|c|}{ Average family size $(n)$} \\
\hline$>3 \cdot 5$ & Ref. & \\
\hline$\leqslant 3 \cdot 5$ & $2 \cdot 45(1 \cdot 64-3 \cdot 08)$ & $<0 \cdot 001$ \\
\hline \multicolumn{3}{|c|}{ Median income (US\$) } \\
\hline$\leqslant 20100$ & Ref. & \\
\hline $20101-30900$ & $1 \cdot 67(1 \cdot 27-2 \cdot 19)$ & $<0.001$ \\
\hline$>30901$ & $1 \cdot 72(1 \cdot 26-2 \cdot 34)$ & $<0.001$ \\
\hline \multicolumn{3}{|l|}{ Higher education } \\
\hline$\leqslant 30 \%$ & Ref. & \\
\hline$>30 \%$ & $1 \cdot 70(1 \cdot 34-2 \cdot 16)$ & $<0.001$ \\
\hline \multicolumn{3}{|l|}{ White } \\
\hline$\leqslant 50 \%$ & Ref. & \\
\hline$>50 \%$ & $1 \cdot 65(1 \cdot 17-2 \cdot 34)$ & $0 \cdot 005$ \\
\hline \multicolumn{3}{|l|}{ Home ownership } \\
\hline$\leqslant 50 \%$ & Ref. & \\
\hline$>50 \%$ & $1 \cdot 29(1 \cdot 03-1 \cdot 62)$ & $0 \cdot 03$ \\
\hline \multicolumn{3}{|c|}{ Living with relatives } \\
\hline$\leqslant 80 \%$ & Ref. & \\
\hline$>80 \%$ & $1 \cdot 63(1 \cdot 30-2 \cdot 03)$ & $<0.001$ \\
\hline
\end{tabular}

IRR, Incidence rate ratio; CI, confidence interval.

Table 2. Census tract factors associated with reported cases of Campylobacter in the 50-59 years age group

\begin{tabular}{|c|c|c|}
\hline Variable & $\begin{array}{l}\text { IRR }(95 \% \mathrm{CI}) \text {, } \\
\text { adjusted for all } \\
\text { other variables }\end{array}$ & $P$ value \\
\hline \multicolumn{3}{|c|}{ Average family size $(n)$} \\
\hline$>3 \cdot 5$ & Ref. & \\
\hline$\leqslant 3 \cdot 5$ & $1 \cdot 52(1 \cdot 11-2 \cdot 08)$ & $0 \cdot 009$ \\
\hline \multicolumn{3}{|c|}{ Median income (US\$) } \\
\hline 20100 & Ref. & \\
\hline $20101-30900$ & $1.43(1.05-1.94)$ & $0 \cdot 02$ \\
\hline$>30901$ & $2 \cdot 00(1 \cdot 45-2 \cdot 77)$ & $<0 \cdot 001$ \\
\hline \multicolumn{3}{|l|}{ Higher education } \\
\hline$\leqslant 30 \%$ & Ref. & \\
\hline$>30 \%$ & $1 \cdot 40(1 \cdot 15-1 \cdot 71)$ & $<0 \cdot 001$ \\
\hline \multicolumn{3}{|l|}{ White } \\
\hline$\leqslant 50 \%$ & Ref. & \\
\hline$>50 \%$ & $2 \cdot 78(1 \cdot 86-4 \cdot 16)$ & $<0.001$ \\
\hline \multicolumn{3}{|c|}{ Living with relatives } \\
\hline$\leqslant 80 \%$ & Ref. & \\
\hline$>80 \%$ & $1 \cdot 31(1 \cdot 09-1 \cdot 56)$ & $0 \cdot 004$ \\
\hline
\end{tabular}

IRR, Incidence rate ratio; CI, confidence interval. limited so that residents rely on processed or fast foods) as a factor but found no association.

In summary, in both the 20-29 and 50-59 years age groups, several census tract factors related to higher socioeconomic status and living with relatives were associated with higher reported rates of campylobacteriosis in Illinois.

\section{Declaration of Interest}

None.

\section{References}

1. Spencer SEF, et al. The spatial and temporal determinants of campylobacteriosis notifications in New Zealand, 2001-2007. Epidemiology and Infection 2011; 138: $1-15$.

2. FoodNet Reports CDC FoodNet. (http://www.cdc.gov/ foodnet/reports.htm). Accessed July 2011.

3. Baker MG, Sneyd E, Wilson NA. Is the major increase in notified campylobacteriosis in New Zealand real? Epidemiology and Infection 2007; 135: 163.

4. Hopkins RS, Olmsted RN. Campylobacter jejuni infection in Colorado: unexplained excess of cases in males. Public Health Reports 1985; 100: 333-336.

5. Manaseki, S. Ethnic inequalities in Campylobacter infection in Birmingham, UK: descriptive study of notified cases. Journal of Epidemiology \& Community Health 2004; 58: 278-279.

6. Miller G, et al. Does age acquired immunity confer selective protection to common serotypes of Campylobacter jejuni? BMC Infectious Diseases 2005; 5: 66.

M. PYRA ${ }^{1}$, MEd, C. CONOVER ${ }^{2}$, MD, MPH, J. HOWLAND ${ }^{2}$, MPH, K. SOYEMI ${ }^{2}, \mathrm{MD}, \mathrm{MPH}$

${ }^{1}$ University of Illinois at Chicago, Chicago, IL, USA

${ }^{2}$ Illinois Department of Public Health, Springfield, IL, USA

Address correspondence to:

Maria Pyra, M.Ed., Research Assistant, University of Illinois at Chicago, 1603 W Taylor St, Chicago, IL, 60607, USA.

(Email:mpyra2@uic.edu) 